\title{
Tin-Oxide-Nanowire-Based Electronic Nose Using Heterogeneous Catalysis as a Functionalization Strategy
}

\author{
Jeong Min Baik, ${ }^{+, \neq}$Mark Zielke, ${ }^{\S}$ Myung Hwa Kim, ${ }^{\dagger, \perp}$ Kimberly L. Turner, ${ }^{\S}$ Alec M. Wodtke, ${ }^{\dagger}$ and \\ Martin Moskovits ${ }^{\dagger, *}$ \\ ${ }^{\dagger}$ Department of Chemistry \& Biochemistry, University of California, Santa Barbara, California 93106, `School of Mechanical and Advanced Materials Engineering, Ulsan \\ National Institute of Science and Technology (UNIST), 100 Banyeon-ri, Eonyang-eup, Ulju-gun, Ulsan, Republic of Korea, 689-805, ${ }^{5}$ Department of Mechanical \\ Engineering, University of California, Santa Barbara, California 93106, and ${ }^{\perp}$ Department of Chemistry \& Nano Science, Ewha Womans University, Seoul, 120-750, Korea
}

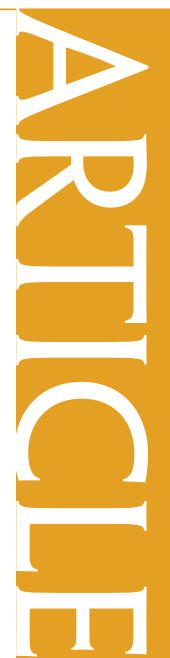

C hemical and biological sensor systems have a profound influence on personal safety, medical diagnosis, detection of pollutants and toxins, and in the transportation industries. ${ }^{1-3}$ Because of their ubiquity and utility a wide range of sophisticated sensors have been developed over a generation; nevertheless, new sensor strategies of ever increasing sensitivity, selectivity, and lower cost seem to be constantly in demand. ${ }^{4-10}$

The ideal chemical or biosensor is a lockand-key system, specific to each target analyte. In the absence of intereferents, the signal intensity from the detector reveals unequivocally the presence and concentration of the targeted analyte. The search for specific recognition agents for key biomarkers is a vibrant and large research field, ${ }^{11}$ with the goal of discovering single or groups of biomarkers with which individual biomolecules, organelles, cells, and microbes can be identified unequivocally signaling, for example, the presence or propensity for disease or potential hazards. Not many such highly specific receptors are yet known, and specific molecular recognition agents for small molecules are even less plentiful.

In contrast, the olfactory receptors in higher mammals are not highly selective-a given receptor responds to many odorants, and a given odorant elicits a response from many olfactory receptors. Nevertheless, odorant identity and concentration can be determined by encoding the interactions of odorants with receptors combinatorially rather than specifically. The pattern of responses is then used by the cognitive apparatus (functioning like piece of software) to classify, identify, and in certain cases quanwww.acsnano.org
ABSTRACT An electronic nose (e-nose) strategy is described based on $\mathrm{SnO}_{2}$ nanowire arrays whose sensing properties are modified by changing their operating temperatures and by decorating some of the nanowires with metallic nanoparticles. Since the catalytic processes occurring on the metal nanoparticles depend on the identity of the metal, decorating the semiconducting nanowires with various metal nanoparticles is akin to functionalizing them with chemically specific moieties. Other than the synthesis of the nanowires, all other steps in the fabrication of the e-nose sensors were carried out using top-down microfabrication processes, paving the way to a useful strategy for making low cost, nanowire-based e-nose chips. The sensors were tested for their ability to distinguish three reducing gases $\left(\mathrm{H}_{2}, \mathrm{CO}\right.$, and ethylene), which they were able to do unequivocally when the data was classified using linear discriminant analysis. The discriminating ability of this e-nose design was not impacted by the lengths or diameters of the nanowires used.

KEYWORDS: electronic nose $\cdot$ nanowire sensor $\cdot$ tin oxide $\cdot$ catalysis $\cdot$ metal nanoparticles

tify, the odorant of interest. Many groups have successfully demonstrated biomimetic electronic and optical versions of this approach over the past two decades, utilizing a variety of multicomponent array strategies to create "electronic noses: e-noses". ${ }^{12-15}$

In most sensors surface-chemical processes are transduced into measurable signals. For example, chemisorptions that result in charge exchange between the adsorbate and its substrate might produce a conductivity change in the substrate. The advent of MEMS microfabrication techniques ${ }^{16}$ and nanotechnology has significantly altered the e-nose paradigm. Because of their very high surface-to-volume ratios, nanoparticles and nanowires can in principle be used to create very sensitive sensors leading to e-nose designs with enhanced sensitivities. ${ }^{17}$

One issue that impacts on the scale-up of nanostructured sensors is the fact that most nanostructures are fabricated by

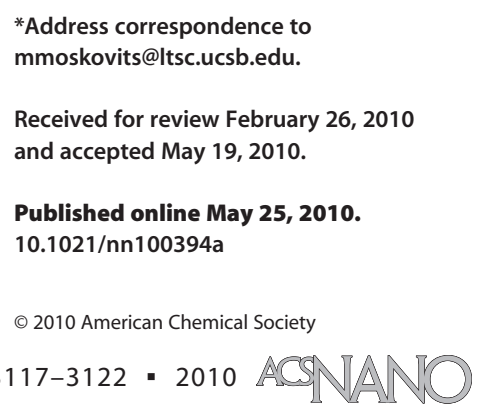




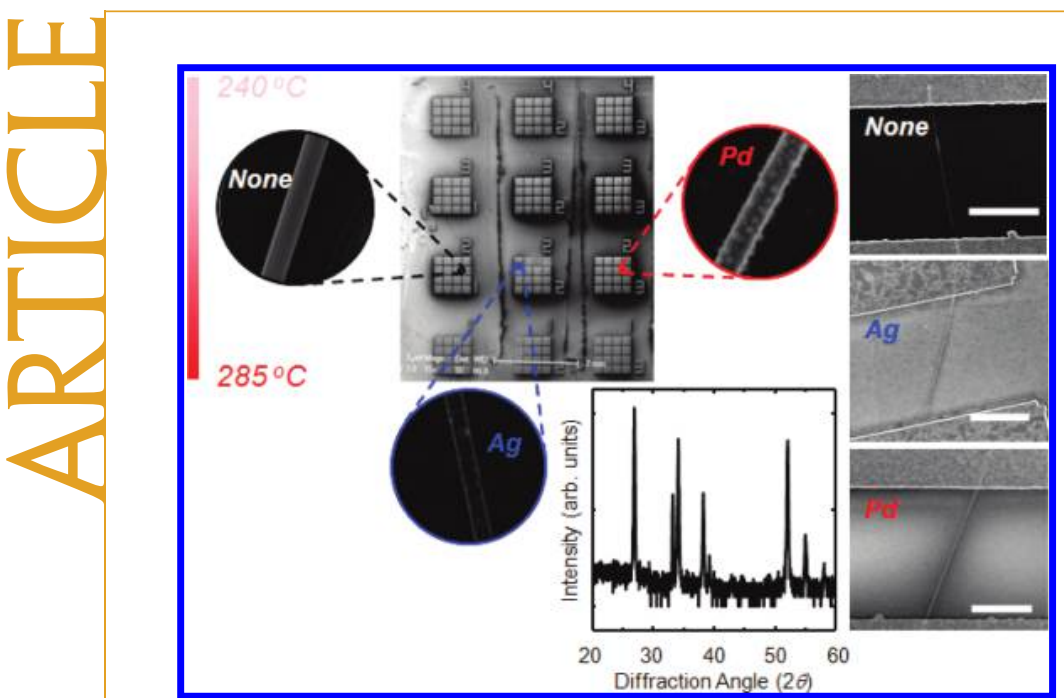

Figure 1. (Upper left) SEM image of the electronic nose device showing the wire-bonding pads, which connect $\mathrm{SnO}_{2}$ nanowires. A meander heater is incorporated into the chip at the bottom producing a 240-285 ${ }^{\circ} \mathrm{C}$ temperature gradient in the vertical direction. SEM images of representative nanowires (pristine, Pd-decorated, and Ag-decorated) are also shown as insets, as well as lower magnification images of the nanowires (right-hand side) to show the manner in which they contact the pads.

The scale bars are $10 \mathrm{~nm}$. A representative XRD of as-grown $\mathrm{SnO}_{2}$ nanowires is also shown.

bottom up technologies which often require the development of innovative post fabrication strategies such as orienting nanowires, forming appropriate electrical contacts, and other processes that simultaneously encompass both the micro- and the nanoscales.

In this paper we describe a strategy for creating an e-nose, which minimizes the bottom up constituent steps and makes choices that are mindful of their integration with top down technologies. For example, we use only a single nanowire platform, $\mathrm{SnO}_{2}$ nanowires (other equally good (single) materials choices could, of
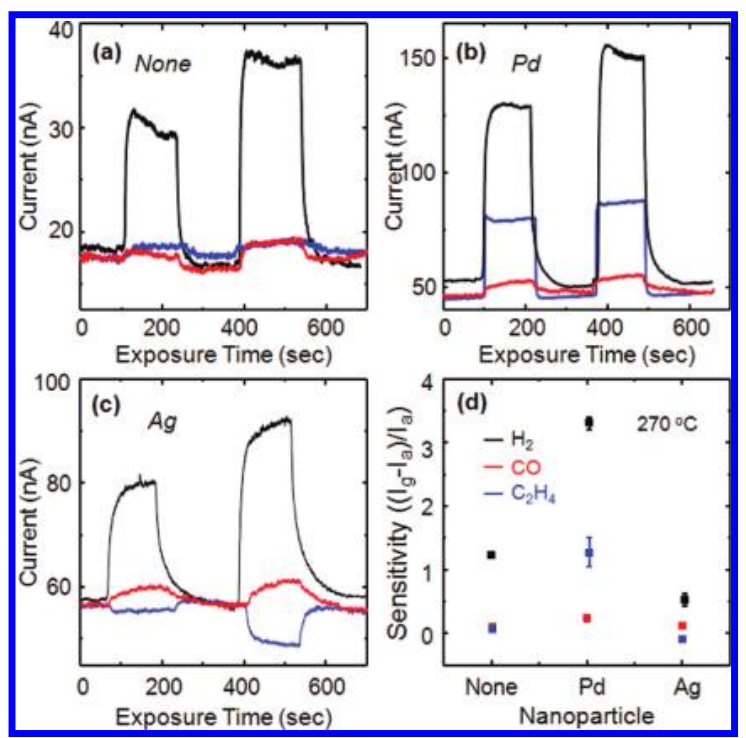

Figure 2. Current responses of (a) a pristine, (b) a Pddecorated, and (c) a Ag-decorated $\mathrm{SnO}_{2}$ nanowire sensor toward $\mathrm{H}_{2}, \mathrm{CO}$, and ethylene measured at $270{ }^{\circ} \mathrm{C}$ in a stream of flowing air (10 sccm). Gas flow rates were 5 and $10 \mathrm{sccm}$. The sensitivities (as defined in the text) deduced from these current differentials are summarized in panel $d$. course, have been made). The nanowires are functionalized only with a variety of metal nanoparticles rather than molecular functionalities because metal deposition through masks is a foundry-friendly process, and heterogeneous catalytic processes show a range of molecular specificity reminiscent of lock-and-key-based molecular recognition. Continuing the catalysis theme, we also operate individual sets of nanowires at various local temperatures on the micrometer scale. The use of a single nanowire, as opposed to several types of nanowires, removes the need to segregate nanowires on a microscopically local scale, a difficult process to scale up for wafer-based manufacturing. Contrariwise, a mat of randomly disposed nanowires can be easily and (statistically) reproducibly laid down at the wafer scale, with all subsequent steps, including producing MEMS-based local heating elements, being carried out and replicated using routine foundry techniques.

Functionalizing metal-oxide nanowires with metal nanoparticles has been widely reported. ${ }^{17-21}$ And by using metal-oxide decorated nanowires one can exploit the vast knowledge amassed over a century of research into heterogeneous catalysis augmented by the fact that nanowires are both nanostructured and pseudoone-dimensional. Accordingly, the sensor can make use of both a very large catalog of well-known surfacechemical processes as well as the changes in the nanowire's conductance, reflecting the surface chemistry occurring both on the surface of the oxide and on the surface of the metal nanoparticle, and processes affecting charge depletion at the metal/oxide Schottky junction. ${ }^{17}$

The e-nose we describe is a two-dimensional microarray of nanowires connected by contacts comprising sets of "receptors" orthogonally varying in their properties on account of two response-modifying strategies. Along one axis the metal-nanoparticle functionality on the tin-oxide nanowires is varied (pristine (no metal), Pd and Ag nanoparticle decoration); while the temperature is varied along an orthogonal axis.

\section{RESULTS AND DISCUSSION}

Surface Functionalization. An SEM image of the chip surface used in this study is shown in Figure 1, with highermagnification SEM images of individual $\mathrm{SnO}_{2}$ nanowires functionalized by nanoparticles shown as insets. SEM imaging indicated that the diameters of the nanoparticles lay in the range 5-22 $\mathrm{nm}$ for $\mathrm{Pd}$ and 9-28 $\mathrm{nm}$ for Ag.

Sensitivity with Metal Nanoparticles. A representative set of source-drain currents, $I_{S D}$, measured as a function of time at an applied source-drain voltage of $1 \mathrm{~V}$ is shown in Figure 2 panels a, b, and c for pristine, $\mathrm{Pd}$ decorated, and Ag-decorated $\mathrm{SnO}_{2}$. One of the three reducing gases was periodically added to the continuously flowing air stream and then shut off. Representative values of the sensitivities of the sensor 
toward the gases are given in Figure $2 \mathrm{~d}$. The sensitivity is defined as $S=\left(I_{\mathrm{g}}-I_{\mathrm{a}}\right) / I_{\mathrm{a}}$, in which $I_{\mathrm{g}}$ and $I_{\mathrm{a}}$ are, respectively, the steady-state current values measured with the analyte gas added to the air stream and just the air stream alone. For nanoparticle-free (pristine) $\mathrm{SnO}_{2}$ nanowires, adding each of the three reducing gases to the air stream causes the source-drain current to increase in less than $1 \mathrm{~min}$, returning to its initial value reversibly when the reducing gases are shut off. For hydrogen, carbon monoxide, and ethylene, respectively, the measured $S$ values on pristine nanowires at $270{ }^{\circ} \mathrm{C}$, were $1.20,0.15$, and 0.12 , with the analyte gas flow rate of $10 \mathrm{sccm}$.

The behavior of all three gases toward $\mathrm{SnO}_{2}$ was consistent with their being reducing agents (i.e., as electron donors). Hydrogen is known to be reactive toward many of the surface sites present on the surface of $\mathrm{SnO}_{2}$, forming species such as $\mathrm{OH}$ groups and water. ${ }^{22} \mathrm{CO}$ has been reported to reduce $\mathrm{SnO}_{2}$ in two ways. It reacts with ionicly chemisorbed surface oxygen to form $\mathrm{CO}_{2}$, and ultimately with lattice oxygens of the oxide to form the same product, much of which desorbs. The reaction with bulk tin oxide can irreversibly reduce the oxide. ${ }^{23}$ These processes lead to increased current resulting from molecule-to-nanowire electron donation.

We find the dependence of the current on gas partial pressure to be approximately linear, although this need not be so in general, since the equilibrium adsorbate surface coverage is related to its partial pressure in the gas phase by an appropriate isotherm which is in general not a linear function of partial pressure. Moreover, the electrical conductance through the nanowire will reflect the surface coverage modulated by the charge transfer that accompanies the surface chemical processes occurring over the time of the measurement, which need not be proportional to the partial pressure (although in general they will be monotonic functions of the partial pressure).

When decorated with $\mathrm{Pd}$, the sensitivities toward hy drogen, carbon monoxide, and ethylene (at $270{ }^{\circ} \mathrm{C}$ ) increase to $3.30,0.25$, and 1.25 , respectively, and the response time is reduced. As reported previously, the large increase in sensitivity toward hydrogen is ascribed to the catalytic dissociation of diatomic hydrogen on, and permeation of atomic $\mathrm{H}$ into, the $\mathrm{Pd}$ nanoparticles. Hydrogen atoms have also been postulated to spill over the nanowire's surface, and diffuse into the tin oxide, further increasing the current. $\mathrm{Pd}$ also provides sites at which $\mathrm{CO}$ and, to some extent, $\mathrm{C}_{2} \mathrm{H}_{4}$ are oxidized. $^{24-26}$

Surface decoration with Ag nanoparticles leads to more complex changes in sensitivity toward the three reducing gases, which also depends on the silver nanoparticle surface density (Figure 2c). Specifically, at 270 ${ }^{\circ} \mathrm{C}$ ethylene causes the current to decrease slightly (10-20\%) below the steady-state value in air. This ob-

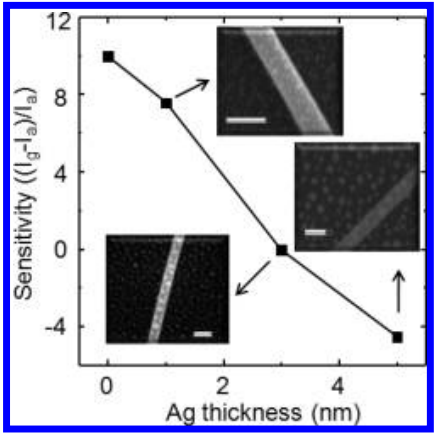

Figure 3. The sensitivity of an $\mathrm{Ag}$-decorated $\mathrm{SnO}_{2}$ nanowire toward ethylene measured at $270{ }^{\circ} \mathrm{C}$ in a flowing oxygen atmosphere as a function of Ag film (mass) thickness. The deposited film breaks up and the metal agglomerates into $\mathrm{Ag}$ nanoparticles. (Inset) Representative SEM images of the Ag nanoparticles decorating the $\mathrm{SnO}_{2}$ nanowire. The scale bars are $100 \mathrm{~nm}$.

servation was previously reported ${ }^{21}$ and ascribed to catalytic ethylene oxidation on Ag, reducing the oxygen concentration on the silver nanoparticles, which, in turn, affects the charge depletion taking place at the Ag-nanoparticle/tin oxide nanowire Schottky junction. When nanowires are functionalized with silver nanoparticles and exposed to hydrogen and $\mathrm{CO}$, the current increases, but with a significantly reduced sensitivity, which decreases to 0.5 and 0.1 , respectively. This is consistent with the reduced surface available for charge exchange, making $\mathrm{Ag}$, unlike $\mathrm{Pd}$, unhelpful toward those gases.

In contrast, the response of the Ag-nanoparticledecorated nanowire to ethylene (introduced into 10 sccm flowing oxygen) was found to depend on the Ag mass thickness used, which translates into the nanoparticle density and average size (Figure 3 ).

For a pristine $\mathrm{SnO}_{2}$ nanowire at $270{ }^{\circ} \mathrm{C}$, the current increases when $10 \mathrm{sccm}$ ethylene gas was added to the background flowing oxygen (Figure 2a). Ag decoration reduces the magnitude of these current increases, and of the resulting sensitivity values, which decrease more or less linearly with Ag mass thickness, becoming negative for Ag film mass thicknesses equal to $\sim 3 \mathrm{~nm}$ and greater. Ag nanoparticles are known to become saturated with chemisorbed oxygen at appropriate values of the ambient temperature and oxygen partial pressure. The introduction of ethylene leads to ethylene epoxidation and the competing reaction of combustion to $\mathrm{CO}_{2}$ and water. ${ }^{27}$ The switch from positive to negative current differentials on exposure to ethylene according to the size and density of the silver nanoparticles decorating the $\mathrm{SnO}_{2}$ nanowire signals a competition between two or more processes occurring concurrently on the silver decorated nanowire. One process is likely the ethylene oxidation, which occurs almost exclusively on the silver surface, the other is likely ethylene chemisorption at $\mathrm{SnO}_{2}$ defects, which produces positive current differentials. This observed dependence of the ethylene response on the Ag surface den- 


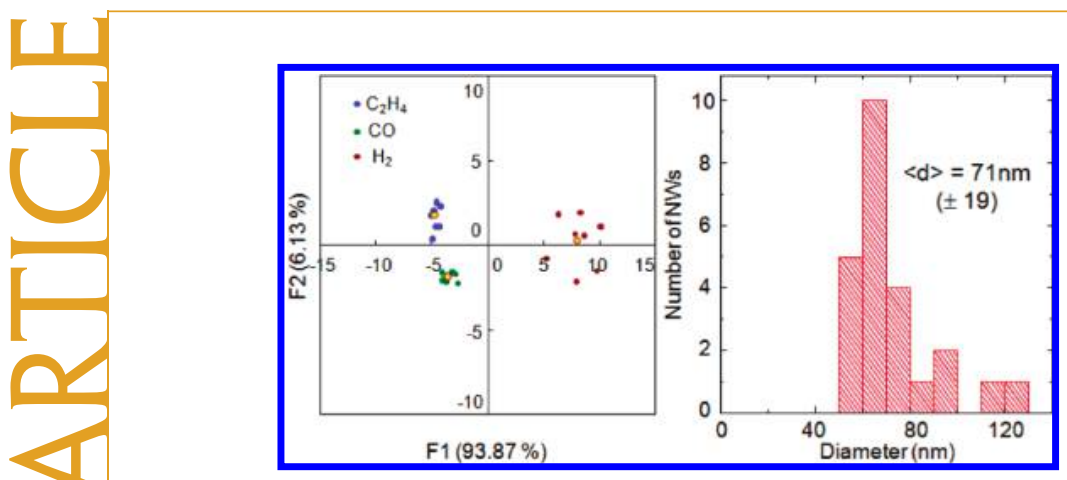

Figure 4. (a) The results of an LDA analysis of the sensitivities obtained for an electronic nose consisting of an array operating with pristine and metal-nanoparticle-decorated $\mathrm{SnO}_{2}$ nanowires, and a $240-285^{\circ} \mathrm{C}$ temperature gradient exposed in turn to 10 sccm flowing $\mathrm{H}_{2}, \mathrm{CO}$, and ethylene in a background stream of air (10 sccm). The LDA analysis classified and separated the points corresponding to the various gases at a $95 \%$ confidence level. (b) Histogram of nanowire diameters (based on SEM imaging) used in the e-nose. The average diameter was $71 \mathrm{~nm}$ with a distribution width of $19 \mathrm{~nm}$.

sity could be used to advantage to further modify the nanowire's sensing properties.

Linear Discriminant Analysis. Steady state current differentials were collected from the 12 sensor elements of the e-nose shown in Figure 1, when exposed to various flows of each of the three test gases. The 12 data sets correspond to the responses of the three classes of nanowires (unfunctionalized and functionalized with $\mathrm{Pd}$ and Ag nanoparticles) at each of four temperature values. The aggregate data set for the e-nose was processed using linear discriminant analysis (LDA) ${ }^{15}$ and plotted as two-dimensional maps to indicate the extent to which the analysis could separate the response of the e-nose to the three reducing gases into separate

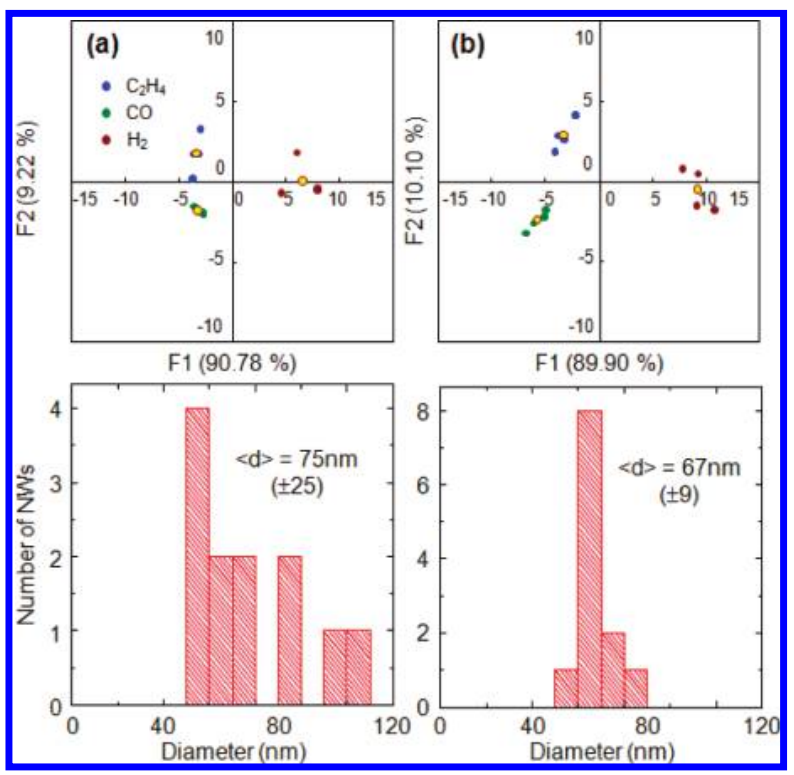

Figure 5. The result of an LDA analysis as in Figure 4 but for two nanowire sets: one with (a) a tight diameter distribution, and (b) a broad distribution of nanowire diameters selected out of the set of nanowires operating in a single e-nose (see the histograms below each LDA plot): (a) average diameter $=67 \mathrm{~nm}$, standard deviation $=9 \mathrm{~nm}$; (b) average diameter $=75 \mathrm{~nm}$, standard deviation $=25 \mathrm{~nm}$. classes. LDA analysis was carried out using a commercial code (xISTAT 2009, Addinsoft, France).

LDA is a method for classifying data into categories by constructing a space mapped by vectors formed from linear combinations of predictor variables (in the present case, the sensitivities derived from the steadystate current differentials of the three nanowire functionalization types at each of the four temperatures, when exposed to one of the three gases). LDA attempts to choose the linear combination that maximizes the distances separating the locations in space of the transformed predictors. The data separation we obtained (Figures $4 \mathrm{a}$ and $5 \mathrm{a}, \mathrm{b}$ ) is very good. That is, the three gases, ethylene, $\mathrm{CO}$, and $\mathrm{H}_{2}$, would be identified unequivocally by the e-nose described here, in gaseous samples each containing one of the three test gases mixed in air. In passing, one is free to choose the variables one uses as predictors. Looking at the data shown in Figure 2, for example, it is clear that aside from the current differentials, one could perform LDA on, for example, the rate constants at which the steady state differentials are reached when the gases are turned on, which qualitatively look quite different for the data subsets.

Electronic Nose Performance with Diameter Distribution. Although, the details of the surface chemistry are sensitive to parameters such as the nanowire's diameter and the degree of metal nanoparticle surface coverage, the ability of a given e-nose to differentiate between the three test gases was found to be independent of such parameters; that is, the overall performance of e-noses fabricated as described above was independent of the nanowires' diameters. That said, samples in which the nanowire diameter distribution was narrower gave tighter point groups in the LDA analysis indicating better analyte discrimination. For example, Figure 5 shows the data recorded for the e-nose chips divided into two sets: (a) average diameter $=75 \mathrm{~nm}$, standard deviation $=25 \mathrm{~nm}$; (b) average diameter $=67 \mathrm{~nm}$, standard deviation $=9 \mathrm{~nm}$. Although both samples give good discrimination in response for the three gases used, the points resulting from the LDA analysis on the data shown in Figure 5b (obtained from nanowires with a narrower distribution of diameters) are somewhat more tightly grouped, indicating better discrimination. (We note in passing that, as with most e-nose strategies, improved precision in identifying specific gases would be expected after subjecting the e-nose to a calibration procedure against known analytes before field use.)

Temperature Dependence of the Sensitivity. The temperature dependence of the sensitivity $(S)$ of an e-nose is shown in Figure 6. The data are presented as $\ln (S)$ vs $1 / T$ to uncover any Arrhenius-like dependence. And indeed for $\mathrm{H}_{2}$ and ethylene, the curves do show fairly good adherence to an expression of the form 


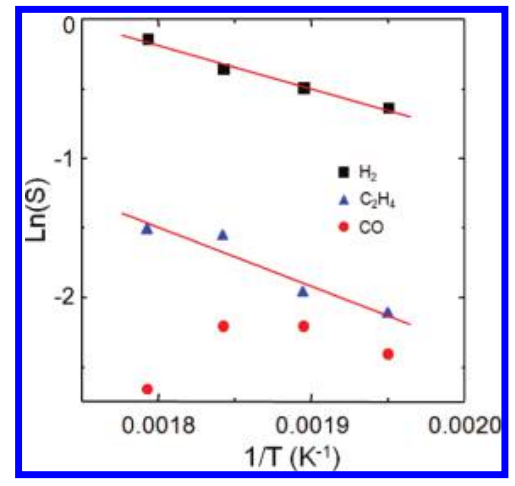

Figure 6. $\ln (S)$ vs $1 / T(K)$ measured for the set of nanowires with the narrow diameter distribution (see Figure 5a) plotted for the three test gases (as shown in the legend). The data for $\mathrm{H}_{2}$ and ethylene follow an Arrhenius-like behavior with energies, respectively, of $\sim 6$ and $\sim 8 \mathrm{kCal} / \mathrm{mol}$. The data for $\mathrm{CO}$ cannot be plotted as a single activated process. Even for the former two gases the linearity of the plot need not imply that a single process is involved but merely that a single equilibrium dominates the many physical processes that affect the response of the e-nose.

$$
S=\exp \left(-\frac{E_{\mathrm{a}}}{k_{\mathrm{B}} T}\right)
$$

with an $E_{\mathrm{a}}$ of $\sim 6$ and $\sim 8 \mathrm{kcal} / \mathrm{mol}$ for $\mathrm{H}_{2}$ and ethylene, respectively. For $\mathrm{CO}$ the temperature dependence of $S$ does not fit an Arrhenius-like equation, likely reflecting the participation of the two aforementioned processes (CO reacting with ionically adsorbed oxygen on the nanowires surface, and with lattice oxygen species). One should point out, however, that the values of $E_{\mathrm{a}}$, varied by almost a factor of 2 when measured for other sets of decorated nanowires, undoubtedly reflecting the fact that the $S$, which is defined in terms of current differentials, depends on multiple parameters, some of which might vary as the physical and geometrical properties of the nanowires used are changed.

In summary, an electronic nose (e-nose) strategy is described which is based on modifying the sensing properties of $\mathrm{SnO}_{2}$ nanowires in two ways: by changing their operating temperatures and by decorating some of the nanowires with metallic nanoparticles. (Pd and $\mathrm{Ag}$ are used in this study.) The nanoparticle decoration affects the surface chemistry occurring on the nanowires and on the metal nanoparticles, and hence their sensing properties in several ways: (i) by creating a charge depletion zone at the Schottky junction formed at the metal/semiconductor interface, which is, in turn, affected by the catalytic processes occurring at the surface of the nanoparticles, and (ii) by the spillover of products of catalysis onto the $\mathrm{SnO}_{2}$ surface. Since catalysis depends critically on the identity of the metal, decorating the semiconducting nanowires with various metal nanoparticles is akin to functionalizing them with chemically specific moieties. Other than the synthesis of the nanowires, all other steps in the fabrication of the e-nose sensors were carried out using topdown microfabrication processes, paving the way toward a useful strategy for making low cost, nanowirebased e-nose chips. The sensors were tested for their ability to distinguish three reducing gases $\left(\mathrm{H}_{2}, \mathrm{CO}\right.$, and ethylene), which they were able to do unequivocally when the data was classified using linear discriminant analysis. Their discriminating ability was not impacted by the lengths or diameters of the nanowires used.

Finally, in principle, a deep understanding of the mechanism whereby the individual sensors operate is unnecessary in demonstrating that one has produced a reliable e-nose, so long as the response pattern is robust, specific, and reproducible; after all, one need not know the mechanism of interaction between an odorant and an individual olfactory receptor to recognize confidently the scent of a rose. Nevertheless, we illustrate the response of the metal-nanoparticle-decorated $\mathrm{SnO}_{2}$ nanowires in some detail (and make reference to prior literature ${ }^{17,21}$ ), in order to support the premise that judiciously chosen heterogeneous catalysts possess the prescribed balance of some, but not overly great, specificity to bestow the required interactive variability to the tin oxide nanowires.

\section{METHODS}

$\mathrm{SnO}_{2}$ Nanowires Synthesis and Characterization. $\mathrm{SnO}_{2}$ nanowires were synthesized by chemical vapor deposition as described previously. ${ }^{21}$ Briefly; an alumina boat with SnO powder (99.9\%, Alfa Aesar) was placed at the center of the quartz tube furnace. A precleaned $\mathrm{SiO}_{2} / \mathrm{Si}$ substrate covered with a $2.0 \mathrm{~nm}$ vapor-deposited Au film was located downstream of the boat. The tube was evacuated, and an Ar carrier gas (50 sccm) containing traces of oxygen were introduced into it and maintained at 130 Torr using a mechanical pump. The furnace temperature was increased to approximately $900^{\circ} \mathrm{C}$, and sample growth proceeded for $1 \mathrm{~h}$, following which the samples were allowed to cool to room temperature in flowing Ar. Field-emission scanning electron microscopy (FEI Sirion XL30-FEG) was used to image the $\mathrm{SnO}_{2}$ nanowires.

SEM imaging indicates that in a typical synthesis, $\mathrm{SnO}_{2}$ nanowires cover the surface of the silicon substrate uniformly and compactly and that the characteristic diameters are in the range of $50-130 \mathrm{~nm}$. One hour of growth typically results in nanowires $\sim 100 \mu \mathrm{m}$ in length, long enough to bridge the 20 $\mu \mathrm{m}$ gap between the electrodes. X-ray diffraction (XRD) analysis of the sample reveals the nanowires to have a tetragonal rutile $\mathrm{SnO}_{2}$ structure with lattice constants $a=4.68 \AA$ and $c=3.15 \AA$.

Electronic Nose Fabrication. The as-synthesized nanowires were transferred by direct contact onto a clean $7 \times 9 \mathrm{~mm}^{2}$ area p-type silicon wafer covered with a $250 \mathrm{~nm}$ thick silica film, which served as an insulating layer. The transfer was carried out by gently pressing the nanowire-covered substrate onto the Si wafer. Altering the pressure during the contact transfer could vary the density in the resulting mat of transferred nanowires, and uniform coverage was obtained over an approximately $1 \mathrm{~cm} \times 1 \mathrm{~cm}$ area of wafer surface. A uniform and reproducible fraction of the nanowires would adhere to the Si wafer following this procedure, which can be automated, and more than one transfer can be carried out from a given mass of nanowires on different Si wafers. Twelve groups of $4 \times 4$ arrays of metallic Ti $(10 \mathrm{~nm}) / \mathrm{Au}$ $(200 \mathrm{~nm})$ micropads were vapor deposited using an appropriate mask to act as source $(S)$ and drain $(D)$ electrodes for the 
nanowires bridging the pads. Samples were annealed in nitrogen for $1 \mathrm{~h}$ at $300{ }^{\circ} \mathrm{C}$ to improve the ohmic contact between the nanowires and metallic micropads. Respectively, 1 and $5 \mathrm{~nm}$ thick (mass thickness) Pd and Ag films were deposited (in serial depositions) using electron beam evaporation at a base pressure of $3.0 \times 10^{-6}$ Torr through a shadow mask appropriately placed over linear sets of four sensor elements to functionalize the surface of the nanowires. (Following annealing, the metal films aggregated into well-separated nanoparticles covering the nanowire uniformly.)

To create the desired temperature profile across the array of gas-sensing segments, each substrate was equipped with a meander-shaped Pt microheater, patterned by conventional lithography which produced a temperature gradient of 240-285 ${ }^{\circ} \mathrm{C}$ over each set of metal-nanoparticle decorated arrays. The morphology of the nanowire layers was inspected by scanning electron microscopy (SEM). To carry out conductometric gassensing tests, the coated chips were mounted in pin-grid-array (PGA) carriers, and wire-bonded.

When the fabrication process was complete each $4 \times 4$ array functioned as one "sensor element"; and each sensor element corresponded to a unique combination of temperature and metal nanoparticle decoration. Of the 24 gaps available in each set of 16-pads corresponding to a given sensor element, several were occupied by nanowires; however, although many gaps did not harbor nanowires, a given gap was rarely bridged by more than one nanowire. For the purposes of the present study two nanowires were chosen for wire-bonding in each of the sensor elements. The process of locating the gaps connected by nanowires and wire bonding their respective pads can be automated, and a greater redundancy of more than 2 nanowires per given set of conditions can be used.

The mounted chips were placed in a reaction chamber and exposed to pulses of one of three reducing gases at various partial pressures entrained in flowing dry air with a flow rate of 10 $\mathrm{sccm}$ : hydrogen $(5-10 \mathrm{sccm})$, ethylene $(5-15 \mathrm{sccm})$, and CO $(5-15 \mathrm{sccm})$.

Three e-nose chips were fabricated using this technique. All three produced similar results and the linear discriminant analysis of each yielded points localized in approximately the same regions of parameter space for the three test gases. The results reported in this report were obtained in a long series of experiments from one of the chips.

Acknowledgment. This work was supported by the Institute for Collaborative Biotechnologies through Grant DAAD19-03-D0004 from the U.S. Army Research Office and made extensive use of the MRL Central Facilities at UCSB supported by the National Science Foundation under Award Nos. DMR-0080034 and DMR0216466 for the HRTEM/STEM microscopy. We gratefully acknowledge the financial support from the Partnership for International Research and Education-for Electronic Chemistry and Catalysis at Interfaces, NSF Grant No. OISE-0530268. Helpful discussions with, and assistance of, Dr. Syed Mubeen are also gratefully acknowledged.

\section{REFERENCES AND NOTES}

1. Kong, J.; Franklin, N. R.; Zhou, C.; Chapline, M. G.; Peng, S.; Cho, K.; Dai, H. Nanotube Molecular Wires as Chemical Sensors. Science 2000, 287, 622-625.

2. Dennis, P. B.; Jaeschke, A.; Saitoh, M.; Fowler, B.; Kozma, S. C.; Thomas, G.; Mammalian, T. O. R. A Homeostatic ATP Sensor. Science 2001, 294, 1102-1105.

3. Kolmakov, A.; Moskovits, M. Chemical Sensing and Catalysis by One-Dimensional Metal-Oxide Nanostructures. Annu. Rev. Mater. Res. 2004, 34, 151-180.

4. Hagleitner, C.; Hierlemann, A.; Lauge, D.; Kimmer, A.; Kerness, N.; Brand, O.; Baltes, H. Smart Single-Chip Gas Sensor Microsystem. Nature 2001, 414, 293-296.

5. Cui, Y.; Wie, Q. Q.; Park, H. K.; Lieber, C. M. Nanowire Nanosensors for Highly Sensitive and Selective Detection of Biological and Chemical Species. Science 2001, 293, 1289-1292.

6. Pan, Z. W.; Dai, Z. R.; Wang, Z. L. Nanobelts of Semiconducting Oxides. Science 2001, 291, 1947-49.
7. Law, M.; Kind, H.; Messer, B.; Kim, F.; Yang, P. D. Photochemical Sensing of $\mathrm{NO}_{2}$ with $\mathrm{SnO}_{2}$ Nanoribbon Nanosensors at Room Temperature. Angew. Chem., Int. Ed. 2002, 41, 2405-2408.

8. Li, C.; Zhang, D. H.; Liu, X. L.; Han, S.; Tang, T.; Han, J.; Zhou, C. W. $\ln _{2} \mathrm{O}_{3}$ Nanowires as Chemical Sensors. Appl. Phys. Lett. 2003, 82, 1613-1618.

9. Wang, H. T.; Kang, B. S.; Ren, F.; Tien, L. C.; Sadik, P. W.; Norton, D. P.; Pearton, S. J.; Lin, J. Hydrogen-Selective Sensing at Room Temperature with ZnO Nanorods. Appl. Phys. Lett. 2005, 86, 243503.

10. Heller, I.; Janssens, A. M.; Mannik, J.; Minot, E. D.; Lemay, S. G.; Dekker, C. Identifying the Mechanism of Biosensing with Carbon Nanotube Transistors. Nano Lett. 2008, 8, 591-595.

11. Dhanasekaran, S. M.; Barrette, T. R.; Ghosh, D.; Shah, R.; Varambally, S.; Kurachi, K.; Pienta, K. J.; Rubin, M. A.; Chinnaiyan, A. M. Delineation of Prognostic Biomarkers in Prostate Cancer. Nature 2001, 412, 822-826.

12. Freund, M. S.; Lewis, N. S. A Chemically Diverse Conducting Polymer-Based Electronic Nose. Proc. Natl. Acad. Sci. U.S.A. 1995, 92, 2652-2656.

13. Gardner, J. W.; Shin, H. W.; Hines, E. L. An Electronic Nose System To Diagnose Illness. Sens. Actuators, B 2000, 70, 19-24.

14. Ampuero, S.; Bosset, J. O. The Electronic Nose Applied to Dairy Products: A Review. Sens. Actuators, B 2003, 94, 1-12.

15. Sysoev, W.; Goschnick, J.; Schneider, T.; Strelcov, E.; Kolmakov, A. A Gradient Microarray Electronic Nose Based on Percolating $\mathrm{SnO}_{2}$ Nanowire Sensing Elements. Nano Lett. 2007, 7, 3182-3188.

16. Cong, H.; Pan, T. Photopatternable Conductive PDMS Materials for Microfabrication. Adv. Funct. Mater. 2008, 18, 1912-1921.

17. Kolmakov, A.; Kelnov, D. O.; Lilach, Y.; Stemmer, S.; Moskovits, M. Enhanced Gas Sensing by Individual $\mathrm{SnO}_{2}$ Nanowires and Nanobelts Functionalized with Pd Catalyst Particles. Nano Lett. 2005, 5, 667-673.

18. Gaidi, M.; Chenevier, B.; Labeau, M. Electrical Properties Evolution under Reducing Gaseous Mixtures $\left(\mathrm{H}_{2}, \mathrm{H}_{2} \mathrm{~S}, \mathrm{CO}\right)$ of $\mathrm{SnO}_{2}$ Thin Films Doped with Pd/Pt Aggregates and used as Polluting Gas Sensors. Sens. Actuators, B 2000, 62, 43-48.

19. Ryzhikov, A. S.; Shatokhin, A. N.; Putilin, F. N.; Rumyantseva, M. N.; Gaskov, A. M.; Labeau, M. Hydrogen Sensitivity of $\mathrm{SnO}_{2}$ Thin Films Doped with Pt by Laser Ablation. Sens. Actuators, B 2005, 107, 387-391.

20. Jain, K.; Pant, R. P.; Lakshmikumar, S. T. Effect of Ni Doping on Thick Film $\mathrm{SnO}_{2}$ Gas Sensor. Sens. Actuators, B 2006, $113,823-829$.

21. Chen, X. H.; Moskovits, M. Observing Catalysis through the Agency of the Participating Electrons: SurfaceChemistry-Induced Current Changes in a Tin Oxide Nanowire Decorated with Silver. Nano Lett. 2007, 7, 807812.

22. Katsuki, A; Fukui, K. $\mathrm{H}_{2}$ Selective Gas Sensor Based on $\mathrm{SnO}_{2}$. Sens. Actuators, B 1998, 52, 30-37.

23. Kolmakov, A.; Zhang, Y.; Cheng, G.; Moskovits, M. Detection of $\mathrm{CO}$ and Oxygen Using Tin Oxide Nanowire Sensors. Adv. Mater. 2003, 15, 997-1000.

24. Stuve, E. M.; Madix, R. J. Oxidation of Ethylene on $\mathrm{Pd}(100)$ - Bonding of Ethylene and Scavenging of Dehydrogenation Fragments by Surface Oxygen. Surf. Sci. 160 1983, 293-304.

25. Duh, J. G.; Jou, J. W.; Chiou, B. S. Catalytic and Gas Sensing Characteristics in Pd-Doped $\mathrm{SnO}_{2}$. J. Electrochem. Soc 1989, 136, 2740-2747

26. Epifani, M.; Arbiol, J.; Pellicer, E.; Comini, E.; Siciliano, P.; Faglia, G.; Morante, J. R. Synthesis and Gas-Sensing Properties of Pd-Doped $\mathrm{SnO}_{2}$ Nanocrystals. A Case Study of a General Methodology for Doping Metal Oxide Nanocrystals. Cryst. Growth Des. 2008, 8, 1774-1778.

27. Linic, S.; Barteau, M. A. Formation of a Stable Surface Oxametallacycle that Produces Ethylene Oxide. J. Am. Chem. Soc. 2002, 124, 310-317. 